\title{
ARTíCULO
}

\section{Análisis de la comunidad del zooplancton gelatinoso (Hydrozoa, Ctenophora, Thaliacea) en el Pacífico central mexicano, abril-mayo 2011}

\author{
Analysis of the gelatinous zooplankton assemblage (Hydrozoa, Ctenophora, Thaliacea) from
} the central Mexican Pacific, April-May 2011

\section{Edgar Gamero-Mora ${ }^{1}$, Gerardo Ceballos-Corona ${ }^{1}$, Rebeca Gasca ${ }^{2}$ y Alejandro Morales-Blake ${ }^{3}$}

\begin{abstract}
'Laboratorio de Biología Acuática, Universidad Michoacana de San Nicolás de Hidalgo, Av. Francisco J. Mujica S/N, Morelia, Michoacán 58030, México. egamero.mora@gmail.com

${ }^{2}$ El Colegio de la Frontera Sur, Unidad Chetumal, Av. Centenario Km. 5.5, A.P. 424, Chetumal, Quintana Roo 77014, México ${ }^{3}$ Facultad de Ciencias Marinas, Universidad de Colima, Carretera Manzanillo-Barra de Navidad Km. 19.5, Manzanillo, Colima 28868, México

Abstract.- The gelatinous zooplankton, an important functional group in the water column, was studied during April and May 2011 in Mexican waters of the Eastern Tropical Pacific. Zooplankton samples and environmental data were obtained during the MareaR-III cruise on board the R/V 'El Puma' (Universidad Nacional Autónoma de México, UNAM). We identified 32 species of gelatinous zooplankton including 25 species of cnidarians (14 medusae, 11 siphonophores), 2 ctenophores and 5 thaliaceans. Up to 14 of these species represent new records for the surveyed area. The medusae Calycopsis simplex, Sphaerocoryne bedoti, Stauridiosarsia baukalion, Lovenella clausa and the siphonophore Sphaeronectes irregularis are recorded for the first time in Mexican waters. High occurrences and/or abundances of the thaliacean Dolioletta gegenbauri, Doliolum nationalis, the medusae Aglaura hemistoma, Liriope tetraphylla, Rhopalonema velatum, Cunina octonaria and the siphonophore Muggiaea atlantica were detected. Lowest diversity values were found in the northern and southern sectors of the surveyed area. The canonical correspondence analysis revealed that most species are related to environmental characteristics of the northern part of the surveyed area. The species richness and the abundance were highly influenced by the occurrence of trachy and narcomedusas in sampling stations both near as far from the shore line, as well as by the occurrence of doliolids. The high abundance of gelatinous zooplankton from oceanic waters throughout the surveyed area is attributed to the narrowness of the continental shelf.
\end{abstract}

Key words: Mexican Pacific, gelatinous zooplankton, pelagic cnidarians, comb jelly, thaliaceans

\begin{abstract}
Resumen.- El zooplancton gelatinoso, un importante grupo funcional en la columna de agua, fue estudiado en aguas mexicanas del Pacífico tropical oriental durante abril y mayo de 2011. El material analizado se recolectó durante la campaña oceanográfica MareaR-III a bordo del B/O 'El Puma' (Universidad Nacional Autónoma de México, UNAM). Se identificaron 32 especies de zooplancton gelatinoso, incluyendo 25 especies de cnidarios (14 medusas, 11 sifonóforos), 2 ctenóforos y 5 taliáceos; 14 son nuevos registros para el área de estudio. Se registran por primera vez en aguas mexicanas las medusas Calycopsis simplex, Sphaerocoryne bedoti, Stauridiosarsia baukalion, Lovenella clausa, y el sifonóforo Sphaeronectes irregularis. Destacan por su frecuencia y por su alta densidad los taliáceos Dolioletta gegenbauri, Doliolum nationalis, las medusas Aglaura hemistoma, Liriope tetraphylla, Rhopalonema velatum, Cunina octonaria y el sifonóforo Muggiaea atlantica. La diversidad de Shannon presentó sus valores menores en las zonas más septentrionales y meridionales. El análisis de correspondencia canónica reveló que la mayoría de las especies son afines a las características ambientales registradas en el sector norte del Pacífico central mexicano. Tanto la riqueza de especies como la densidad de organismos están determinadas por la presencia de narcomedusas y traquimedusas, así como por doliólidos. La alta densidad de seres del zooplancton gelatinoso de aguas oceánicas en toda la zona de estudio es favorecida por la estrechez de la plataforma continental en el Pacífico central mexicano.
\end{abstract}

Palabras clave: Pacifico mexicano, zooplancton gelatinoso, cnidarios pelágicos, ctenóforos, taliáceos 


\section{INTRODUCCIÓN}

El zooplancton gelatinoso es un grupo funcional integrado por organismos de los phyla Cnidaria, Ctenophora y Chordata (Thaliacea) (Morales-Ramírez \& Nowaczyk 2006). Estos grupos comparten un estilo de vida planctónico y un cuerpo frágil y transparente compuesto principalmente por agua (Lebrato \& Jones 2011); ecológicamente, destacan los ctenóforos y los cnidarios por ser eficientes depredadores en la columna de agua, mientras que los taliáceos son filtradores. Las medusas pueden formar densas agrupaciones que cubren vastas áreas en los océanos ocasionando pérdidas económicas en distintos sectores (Alvarez et al. 2003). Por ejemplo, en el ámbito pesquero por sus hábitos depredadores, en el turístico por su capacidad urticante y en el industrial durante sus explosiones demográficas (Purcell 1985, Purcell et al. 2007, Schiariti 2008). Los taliáceos también son capaces de agregarse y contribuir de manera importante al flujo trófico de materia orgánica en la columna de agua (Hereu et al. 2010).

Los organismos del zooplancton gelatinoso pueden representar fuentes de alimento, proteínas, genes o compuestos de interés en neurobiología y medicina (Lucas 2011). Asimismo, pueden ser útiles como indicadores del movimiento de masas de agua (Fagetti 1973) y juegan un papel importante en el ciclo del carbono y la transferencia de energía en los ecosistemas (Bailey et al. 1995, Madin et al. 2006).

Aunque los grupos del zooplancton gelatinoso han sido reportados como ampliamente distribuidos en los mares del mundo (Touzri et al. 2012), se conoce poco acerca de su composición, densidad y distribución en el Pacífico mexicano. En la zona central del Pacífico mexicano la mayoría de los antecedentes provienen de reportes de expediciones internacionales (Maas 1897, Agassiz \& Mayer 1902, Bigelow 1909, 1911, 1912; Foerster 1923, Alvariño 1972), existiendo una reducida cantidad de información específica para la zona de estudio (SeguraPuertas 1984, Segura-Puertas et al. 2010). Además, no han sido estudiados en conjunto como un grupo funcional. En el presente trabajo se cubre una extensa área del Pacífico mexicano, lo cual contribuye al conocimiento sobre el zooplancton gelatinoso en la región del Pacífico Tropical Oriental. Los objetivos incluyeron identificar morfológicamente el zooplancton gelatinoso, estimar su frecuencia de aparición, densidad y diversidad, así como determinar la influencia de los parámetros ambientales en su distribución. Todo ello, a partir de la revisión de muestras de zooplancton de red y datos de variables hidrológicas recolectados desde Cabo Corrientes, Jalisco hasta Punta Diamante, Guerrero en la campaña oceanográfica MareaR-III abordo del B/O 'El Puma' de la Universidad Nacional Autónoma de México (UNAM).

\section{MATERIALES Y MÉTODOS}

Las muestras de zooplancton, así como los registros de las variables físicas, químicas y biológicas se obtuvieron en la campaña oceanográfica MareaR-III, realizada del 23 de abril al 7 mayo de 2011 a bordo del B/O 'El Puma' de la UNAM. Se visitaron 43 estaciones de muestreo distribuidas en las zona nerítica y oceánica del Pacífico central mexicano, frente a la costa de los estados de Jalisco, Colima, Michoacán y Guerrero (Fig. 1). Las muestras de zooplancton se recolectaron mediante arrastres verticales a una velocidad de $30 \mathrm{~m} \mathrm{~s}^{-1}$ con una red estándar con diámetro de boca de $32 \mathrm{~cm}$, una longitud de $116 \mathrm{~cm}$ y una abertura de malla de $202 \mu \mathrm{m}$. Las muestras fueron fijadas con formaldehído al 5\% amortiguado con tetraborato de sodio. El volumen de agua filtrado en cada sitio de muestreo se calculó por el método del cilindro, es decir, multiplicando el área de la boca de la red por la distancia recorrida en el arrastre. Los lances se realizaron a diferentes profundidades (30-160 m) según la ubicación del máximo de clorofila en cada sitio de muestreo. Previo a la recolección del plancton, se obtuvieron en la columna de agua registros continuos de clorofila (fluorescencia de la clorofila), oxígeno disuelto, salinidad y temperatura con un CTD Seabird SBE 9 Plus. Para brindar un panorama

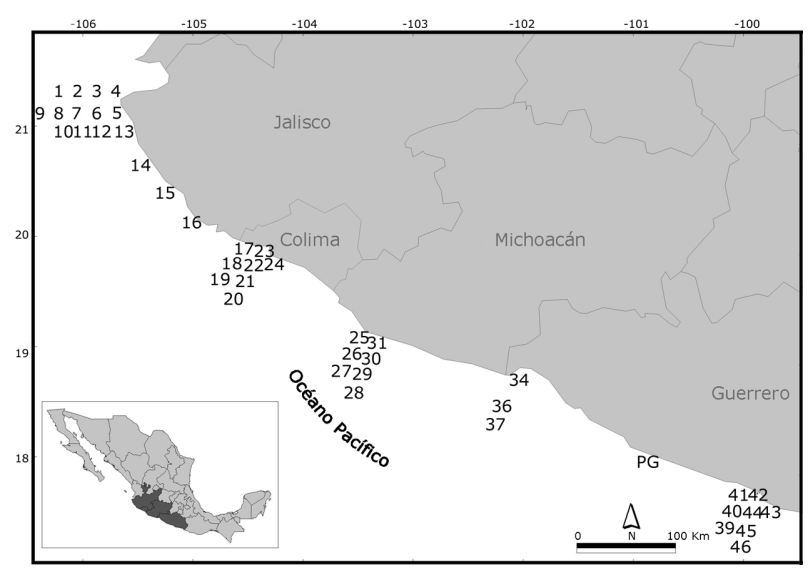

Figura 1. Zona de estudio y ubicación de los sitios de muestreo en el Pacífico central mexicano / Surveyed area and location of sampling sites in the central Mexican Pacific 
general de las condiciones ambientales del área de estudio, se presenta de manera gráfica la información de 4 trayectos (transectos) conformados por distintas estaciones de muestreo: T1 (5, 6, 7, 8, 9), T2 (20, 21, 22, 23), T3 $(28,29,30,31)$ y T4 $(42,44,45,46)$. Los datos de cada trayecto y variable corresponden al promedio de los perfiles verticales del grupo de estaciones que lo conforma. Además, se muestran mapas de la distribución horizontal de las variables hidrológicas elaborados a partir de la media de los registros superficiales de cada punto de recolecta $(0-50 \mathrm{~m})$.

Las muestras se tiñeron con rosa de bengala para facilitar la observación y separación del zooplancton gelatinoso entre el detritus (Collins \& Williams 1981). Los organismos gelatinosos (medusas, sifonóforos, ctenóforos y taliáceos) se separaron de cada muestra original y se identificaron en la mayoría de los casos a nivel especifico con ayuda de los trabajos de Godeaux (1998), Mianzan (1999) y Bouillon et al. (2004). El material analizado, así como las muestras originales se encuentran depositados en el laboratorio de Biología Acuática 'J. Javier Alvarado Díaz’ de la Universidad Michoacana de San Nicolás de Hidalgo, México.

Se utilizó la escala propuesta por Licea (1974) para determinar la frecuencia de aparición de las especies, clasificándolas como predominantes (presentes entre el 80-100\% de las estaciones de muestreo), abundantes (50$79 \%$ ), frecuentes $(20-49 \%)$, presentes $(10-19 \%)$, escasas $(5-9 \%)$, raras $(1-4 \%)$ y excepcionales $(<1 \%)$. Algunas de las muestras se contabilizaron de manera completa, y en las más abundantes se obtuvieron alícuotas de 1/2-1/8 con un fraccionador Folsom (Sánchez \& Ponce 1996). Para contabilizar los sifonóforos calicóforos se contó el número de nectóforos anteriores y posteriores, registrándose el mayor de ambos. En el caso de los fisonectes, cada uno de los nectóforos se consideró un individuo (Pugh 1984). El número de organismos contabilizados se estandarizó a org. $1000 \mathrm{~m}^{-3}$. Se calculó la diversidad de Shannon y la equidad de Pielou.

Se realizó un dendrograma de similitud (Bray-Curtis) por el método de promedio de grupos, en el cual, todas las especies fueron consideradas aplicando la transformación a raíz cuadrada. La significancia estadística de los agrupamientos obtenidos se evaluó con un análisis de perfiles de similitud (SIMPROF). La identificación de las especies que contribuyen en mayor grado a la formación de los grupos se realizó mediante un análisis de porcentajes de similitud (SIMPER) (Clarke \&
Warwick 2001, Clarke \& Gorley 2006).

Los datos biológicos y ambientales fueron estudiados en conjunto mediante un análisis de correspondencia canónica (ACC). Para ello, las densidades se transformaron a raíz cuarta y los registros de las variables ambientales se normalizaron con la fórmula: $\mathrm{Z}=(\mathrm{x}-\overline{\mathrm{X}}) \mathrm{s}^{-1}(\mathrm{Z}$ : valor normalizado; $\mathrm{x}$ : valor a normalizar; $\overline{\mathrm{X}}$ : media de la variable; $\mathrm{s}$ : desviación estándar). No se emplearon los registros de las variables ambientales ni los datos de densidad del sitio de muestreo 42 en el ACC, debido al sesgo que representaban por la alta densidad de organismos encontrados en dicho punto.

\section{Resultados}

\section{VARIables físicas, QUímicas Y BIOLÓGICAS}

La concentración más elevada de clorofila se registró en T1 (21 m; 10,19 $\mathrm{mg} \mathrm{m}^{-3}$ ) y la mínima en T3 (91 m; 0,06 mg $\left.\mathrm{m}^{-3}\right)$. La profundidad en la que se registró el máximo valor de clorofila en cada trayecto varió, estando presente en T1, T2, T3 y T4 a 21, 30, 40 y 44 m, respectivamente. La fluorescencia de la clorofila mostró sus mayores valores en los sitios ubicados en el sector norte del área de estudio (Figs. 2 y 3A). A excepción de T4, las mayores concentraciones de oxígeno disuelto se registraron en profundidades menores a $10 \mathrm{~m}$, en T4 se encontraron tanto los mayores (29 m; 6,12 $\left.\mathrm{ml} \mathrm{L}^{-1}\right)$ como los menores (100 m; $\left.0,14 \mathrm{ml} \mathrm{L}^{-1}\right)$ valores de esta variable. El oxígeno disuelto mostró un aumento en su concentración hacia la parte sur del área de estudio (Figs. 2 y 3B). La salinidad alcanzó su máxima concentración en T4 (84 m; 34,89) y la mínima en T1 (13 m; 34,47), con respecto a su distribución horizontal, las mayores concentraciones se observaron en la zona norte; este parámetro manifestó una mayor homogeneidad entre estaciones costeras y oceánicas de los sectores norte y sur del área de estudio, ya que en la zona central se registraron aguas menos saladas en sitios costeros (Figs. 2 y 3C). En relación con la temperatura, tanto la menor $\left(100 \mathrm{~m} ; 13,85^{\circ} \mathrm{C}\right)$ como la mayor $\left(4 \mathrm{~m} ; 29,56^{\circ} \mathrm{C}\right)$ se registraron en T4. Las mayores temperaturas se encontraron en los puntos de muestreo más meridionales (Figs. 2 y 3D).

\section{COMPOSICIÓN DEL ZOOPLANCTON GELATINOSO}

Se identificaron 32 especies, siendo los cnidarios el componente con mayor riqueza específica. Se registraron 13 especies de hidromedusas (41\%), 11 de sifonóforos (34\%), 1 de escifomedusas (3\%), 5 de taliáceos (16\%) y 2 de ctenóforos $(6 \%)$ (Tabla 1). 


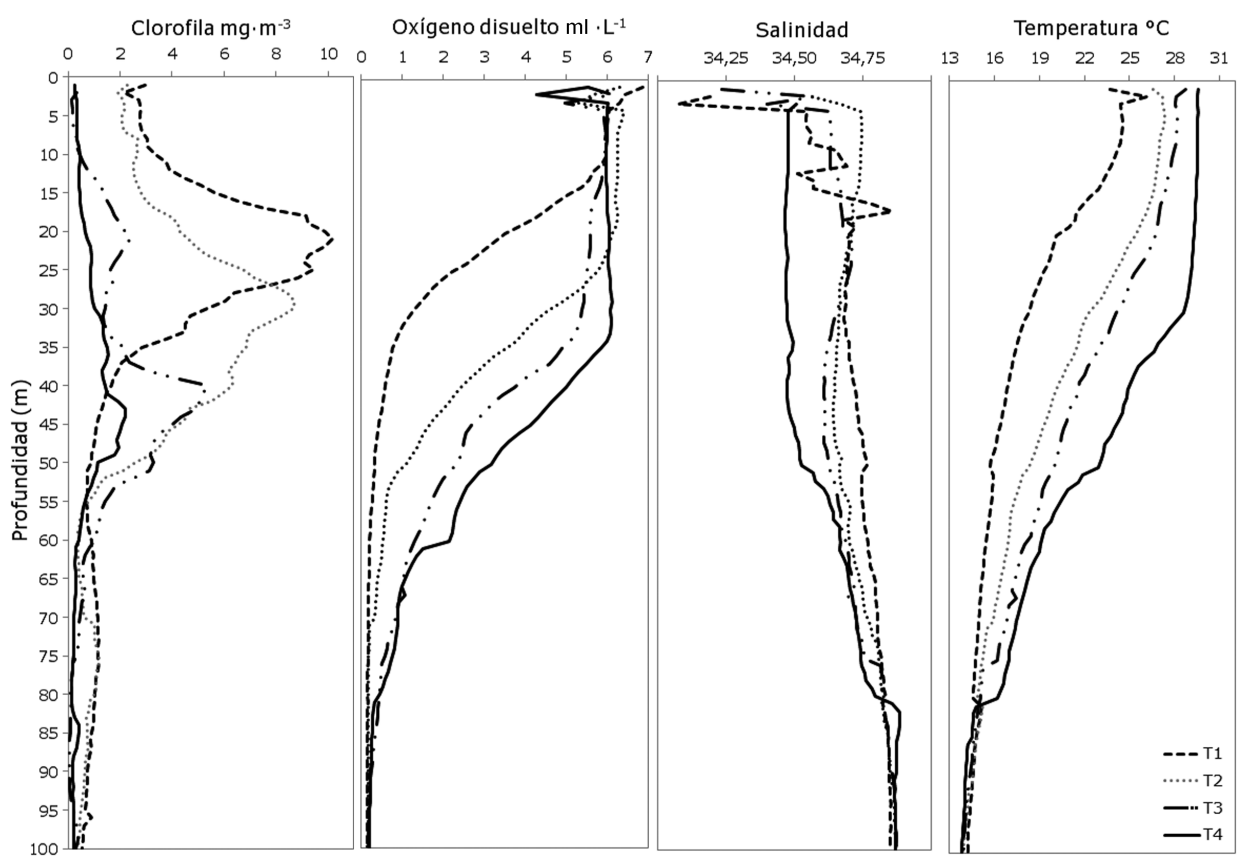

Figura 2. Distribución vertical de la clorofila, oxígeno disuelto, salinidad y temperatura en 4 trayectos de estaciones. $\mathrm{T1}=$ trayecto $1 ; \mathrm{T} 2=$ trayecto 2; T3=trayecto 3; T4=trayecto 4/ Vertical distribution of chlorophyll, dissolved oxygen, salinity and temperature in 4 transects of stations. $\mathrm{T} 1=$ transect $1 ; \mathrm{T} 2=$ transect $2 ; \mathrm{T} 3=$ transect $3 ; \mathrm{T} 4=$ transect 4

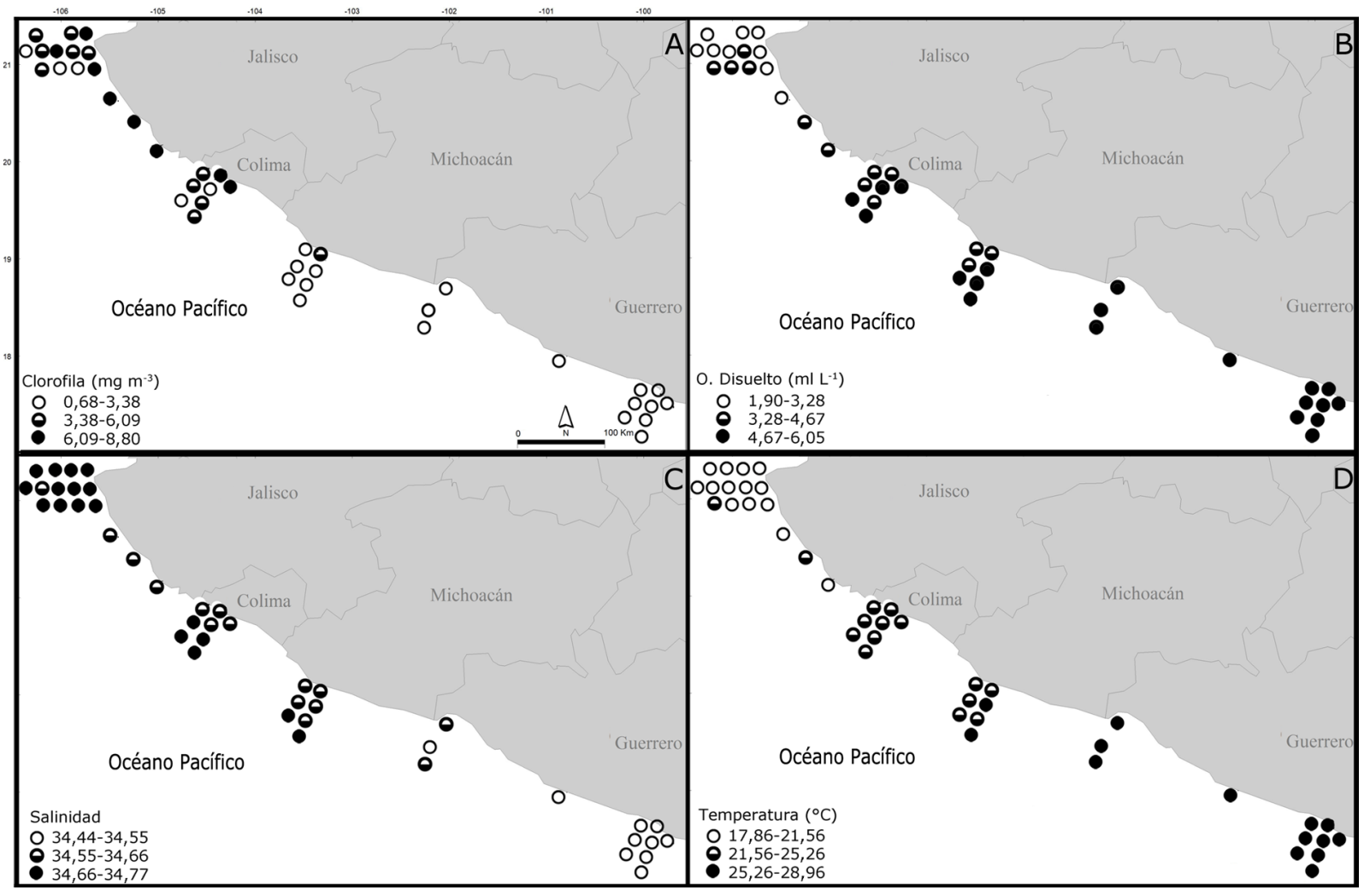

Figura 3. Distribución horizontal de clorofila (A), oxígeno disuelto (B), salinidad (C) y temperatura (D). Cada punto corresponde al promedio de los datos registrados desde la superficie hasta los $\mathbf{5 0 ~} \mathbf{m}$ de profundidad / Horizontal distribution of chlorophyll (A), dissolved oxygen (B), salinity (C) and temperature (D). Each point represents the average of data recorded from the surface to 50 $m$ depth 
Tabla 1. Especies, densidad de organismos y frecuencia de aparición del zooplancton gelatinoso en el área de estudio / Species, organisms densities and occurrence of gelatinous zooplankton in the surveyed area

\begin{tabular}{|c|c|c|c|c|c|c|c|}
\hline \multirow{2}{*}{$\begin{array}{c}\text { Grupo } \\
\text { taxonómico }\end{array}$} & \multirow[b]{2}{*}{ Especie } & \multicolumn{5}{|c|}{ Densidad (org. $1000 \mathrm{~m}^{-3}$ ) } & \multirow{2}{*}{$\begin{array}{l}\text { Frecuencia } \\
\text { de aparición } \\
(\%)\end{array}$} \\
\hline & & Máxima & $\begin{array}{c}\text { Media } \\
\text { Geométrica }\end{array}$ & $\begin{array}{c}\text { Desviación } \\
\text { estándar }\end{array}$ & Total & Relativa & \\
\hline \multicolumn{8}{|l|}{ CNIDARIA } \\
\hline \multirow[t]{6}{*}{ Anthomedusae } & Bougainvillia muscus & 2984 & 107 & 494 & 4621 & 0,14 & 6,98 \\
\hline & Cytaeis tetrastyla & 311 & 7 & 47 & 311 & 0,01 & 2,33 \\
\hline & Calycopsis simplex & 355 & 8 & 54 & 355 & 0,01 & 2,33 \\
\hline & Sphaerocoryne bedoti & 829 & 32 & 150 & 1382 & 0,04 & 4,65 \\
\hline & Stauridiosarsia baukalion & 23210 & 540 & 3539 & 23210 & 0,68 & 2,33 \\
\hline & Porpita porpita & 311 & 7 & 47 & 311 & 0,01 & 2,33 \\
\hline \multirow[t]{2}{*}{ Leptomedusae } & Lovenella clausa & 497 & 19 & 90 & 829 & 0,02 & 4,65 \\
\hline & Obelia sp. & 36473 & 860 & 5561 & 36970 & 1,09 & 4,65 \\
\hline \multirow[t]{2}{*}{ Narcomedusae } & Solmundella bitentaculata & 22381 & 1593 & 3522 & 68493 & 2,01 & 74,42 \\
\hline & Cunina octonaria & 10942 & 1440 & 1909 & 61911 & 1,82 & 86,05 \\
\hline \multirow[t]{11}{*}{ Siphonophorae } & Abylopsis eschscholtzi & 10610 & 943 & 1649 & 40562 & 1,19 & 72,09 \\
\hline & Abylopsis tetragona & 6631 & 678 & 1112 & 29164 & 0,86 & 69,77 \\
\hline & Chelophyes contorta & 1326 & 85 & 239 & 3674 & 0,11 & 18,60 \\
\hline & Diphyes bojani & 1989 & 142 & 351 & 6092 & 0,18 & 23,26 \\
\hline & Diphyes dispar & 3979 & 910 & 954 & 39139 & 1,15 & 76,74 \\
\hline & Eudoxoides mitra & 829 & 87 & 175 & 3755 & 0,11 & 25,58 \\
\hline & Muggiaea atlantica & 53466 & 8780 & 10463 & 377555 & 11,10 & 97,67 \\
\hline & Hippopodius hippopus & 1326 & 169 & 375 & 7262 & 0,21 & 23,26 \\
\hline & Sphaeronectes irregularis & 1326 & 120 & 308 & 5181 & 0,15 & 18,60 \\
\hline & Agalma sp. & 1326 & 76 & 262 & 3254 & 0,10 & 9,30 \\
\hline & Nanomia bijuga & 13263 & 397 & 2066 & 17091 & 0,50 & 9,30 \\
\hline \multirow[t]{3}{*}{ Trachymedusae } & Liriope tetraphylla & 23625 & 2331 & 3969 & 100220 & 2,95 & 88,37 \\
\hline & Aglaura hemistoma & 62999 & 9762 & 13674 & 419764 & 12,34 & 86.05 \\
\hline & Rhopalonema velatum & 7958 & 1436 & 1899 & 61754 & 1,82 & 81,40 \\
\hline Scyphozoa & Pelagia noctiluca & 17905 & 1565 & 4176 & 67305 & 1,98 & 39,53 \\
\hline \multicolumn{8}{|l|}{ CTENOPHORA } \\
\hline Tentaculata & Pleurobrachia pileus & 86209 & 2124 & 13141 & 91320 & 2,68 & 11,63 \\
\hline Nuda & Beroe cucumis & 1326 & 49 & 213 & 2114 & 0,06 & 9,30 \\
\hline \multicolumn{8}{|l|}{ CHORDATA } \\
\hline \multirow[t]{3}{*}{ Salpida } & Pegea confoederata & 1243 & 29 & 190 & 1243 & 0,04 & 2,33 \\
\hline & Thalia democratica & 4974 & 324 & 916 & 13925 & 0,41 & 27,91 \\
\hline & Iasis cylindrica & 5222 & 129 & 797 & 5554 & 0,16 & 4,65 \\
\hline \multirow[t]{2}{*}{ Doliolida } & Dolioletta gegenbauri & 729458 & 27691 & 111380 & 1190733 & 35,00 & 95,35 \\
\hline & Doliolum nationalis & 235416 & 16681 & 39217 & 717271 & 21,08 & 90,70 \\
\hline
\end{tabular}


Frecuencia, densidad, EQUidad y diversidad Del ZOOPLANCTON GELATINOSO

Las especies raras y escasas representaron el mayor porcentaje de individuos encontrados en el área de estudio (41\%), seguido por aquellas que mostraron una frecuencia de aparición continua, es decir, predominantes y abundantes (34\%) y por último las especies frecuentes y presentes que fueron aquellas que aparecieron con cierta periodicidad (25\%). El sifonóforo Muggiaea atlantica, los taliáceos Dolioletta gegenbauri y Doliolum nationalis, y las medusas Liriope tetraphylla, Aglaura hemistoma, Cunina octonaria y Rhopalonema velatum fueron las especies que se recolectaron con mayor frecuencia en el área de estudio (Tabla 1).

Los cnidarios representaron el 40,60\% del total de zooplancton gelatinoso; las medusas A. hemistoma, $L$. tetraphylla y Solmundella bitentaculata y el sifonóforo M. atlantica fueron las especies que presentaron mayores densidades. Por otro lado, los ctenóforos constituyeron el 2,75\% del total, siendo Pleurobrachia pileus el más abundante del grupo. Los taliáceos fueron el taxón más abundante en la zona de muestreo representando en conjunto el $56,69 \%$ de la densidad; $D$. gegenbauri fue la especie más abundante en el área de estudio, alcanzando densidades máximas de 729458 org. $1000 \mathrm{~m}^{-3}$ (Tabla 1).

La mayor densidad de individuos se registró en la estación $42\left(1187028 \pm 134037\right.$ org. $\left.1000 \mathrm{~m}^{-3}\right)$. Por otro lado, la menor fue registrada en el sitio 19 (9201 \pm 606 org. $1000 \mathrm{~m}^{-3}$ ). Los sitios de muestreo 2, 8, 9, 14, 16, 24 y 42 presentaron densidades superiores a los 100000 org. $1000 \mathrm{~m}^{-3}$ (Fig. 4). En todos los casos, el incremento en la densidad de organismos en dichas estaciones

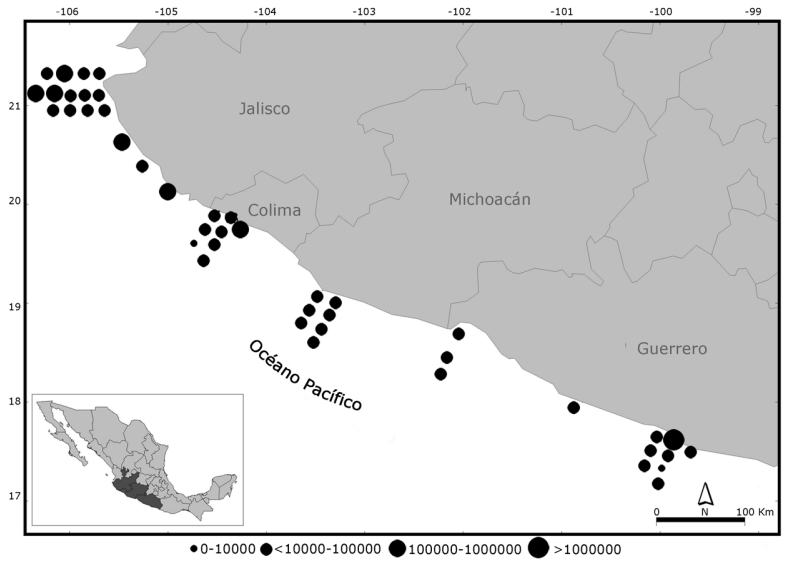

Figura 4. Distribución de las densidades totales de zooplancton gelatinoso en el área de estudio / Distribution of total densities of the gelatinous zooplankton in the surveyed area de muestreo fue debido a las elevadas densidades de $D$. gegenbauri, D. nationalis, A. hemistoma y/o M. atlantica.

La diversidad de Shannon alcanzó un valor máximo de 2,42 bits en la estación 26 y un valor mínimo de 0,88 bits en la estación 7, el valor promedio en el área de estudio fue de 1,87 bits. Por otro lado, 0,92 fue la cifra más elevada de la equidad de Pielou en el sitio 20, 0,36 la menor en la estación 7 y 0,77 la media para la zona de muestreo. La mayor riqueza de especies se registró en la estación 30 y la menor en el sitio 9 (Fig. 5).

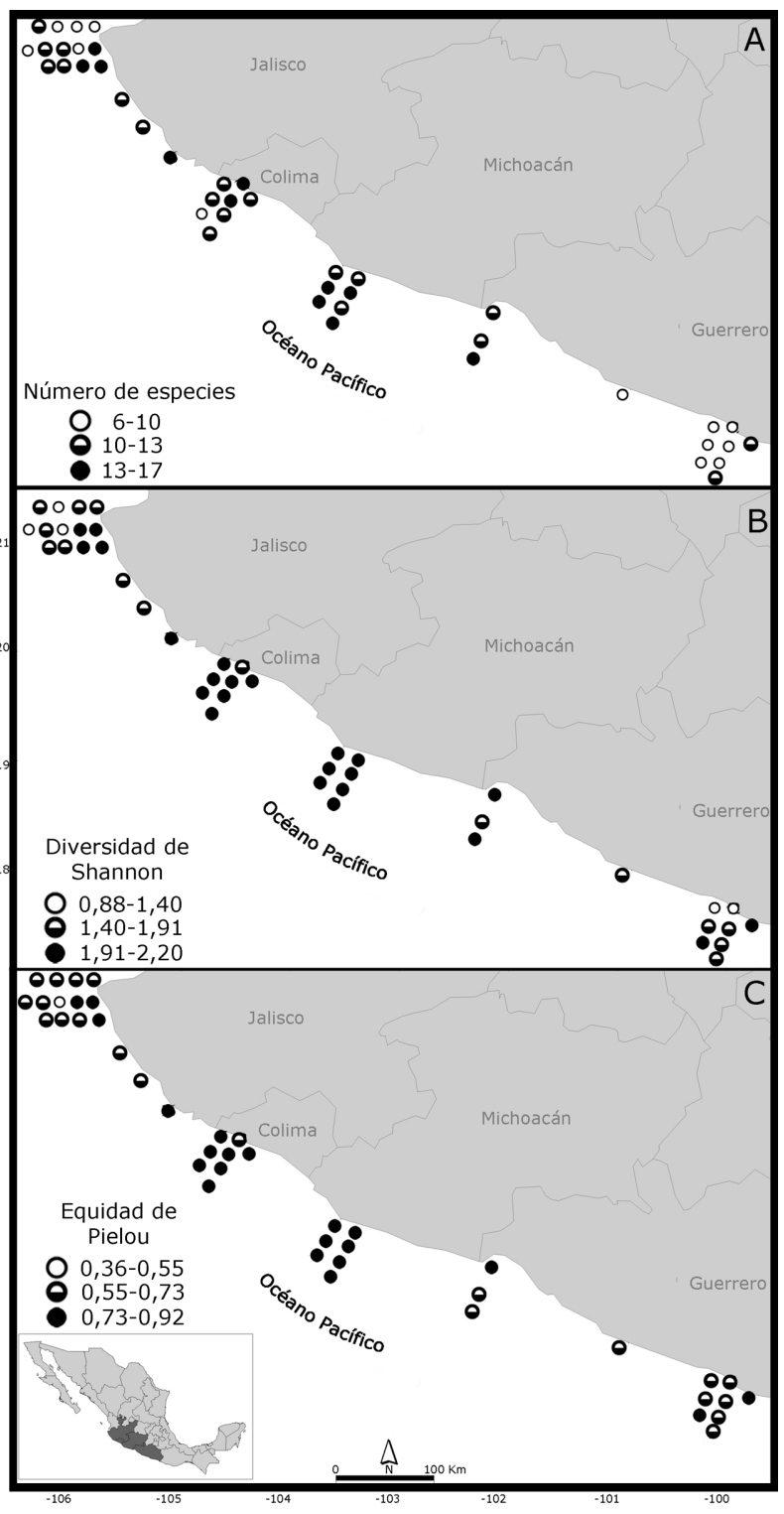

Figura 5. Riqueza específica (A), diversidad de Shannon (B) y equidad de Pielou (C) por sitio de muestreo / Species richness (A), Shannon diversity (B) and Pielou's evenness (C) by sampling sites 


\section{ANÁLISIS DE LA COMUNIDAD}

Se observó la formación de 4 grupos de estaciones con una distribución similar de especies entre ellas (SIMPROF, $P<0,05)$. Los conjuntos I y II estuvieron conformados por estaciones en las cuales el lance de la red se realizó a una profundidad máxima de $75 \mathrm{~m}$, mientras que III y IV por una mezcla de estaciones con lances realizados a profundidades mayores a $75 \mathrm{~m}$ (Fig. 6A). El grupo I estuvo conformado por un par de sitios de muestreo costeros ubicados en el sector sur del área de estudio, el II por estaciones septentrionales, el III por puntos de recolecta distribuidos especialmente en las partes norte y sur del área de estudio y el IV por sitios distribuidos en su mayoría en el sector medio del Pacífico central mexicano (Fig. 6B). La abundancia de las especies influyó de la siguiente manera: el grupo II concentró a las estaciones con las mayores densidades de organismos, seguido por los conglomerados III, I y IV en orden descendente de abundancia (Fig. 7A).

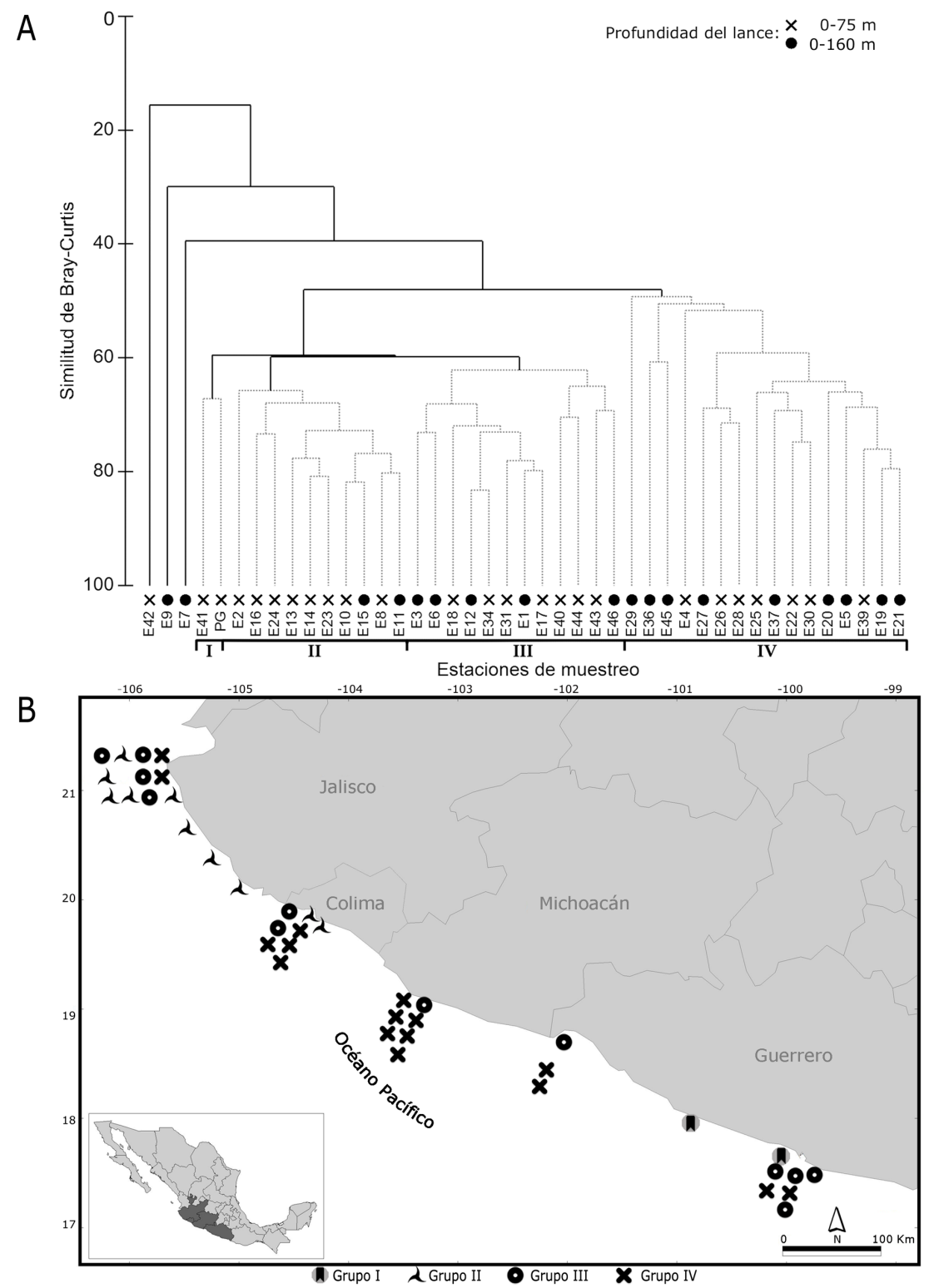

Figura 6. Dendrograma basado en la similitud de Bray-Curtis para los sitios de muestreo (A) y distribución geográfica de los grupos formados en el análisis de similitud (B) / Dendrogram based on the Bray-Curtis similarity index for sampling sites (A) and geographic distribution of the clusters (B) 
Las especies que definieron la formación de los 4 conjuntos fueron Doliolum nationalis, Dolioletta gegenbauri, Aglaura hemistoma y Muggiaea atlantica. Si bien la formación de los grupos estuvo determinada por dichas especies, la diferencia entre ellos radicó en la variación en el porcentaje de contribución de cada una de ellas. De esta manera, $D$. nationalis presentó el mayor porcentaje de contribución en el grupo I, D. gegenbauri en el II, A. hemistoma en el III y M. atlantica en el IV (Fig. 7B). La comparación entre grupos permitió distinguir que aunque con menor influencia, hubo otras especies que

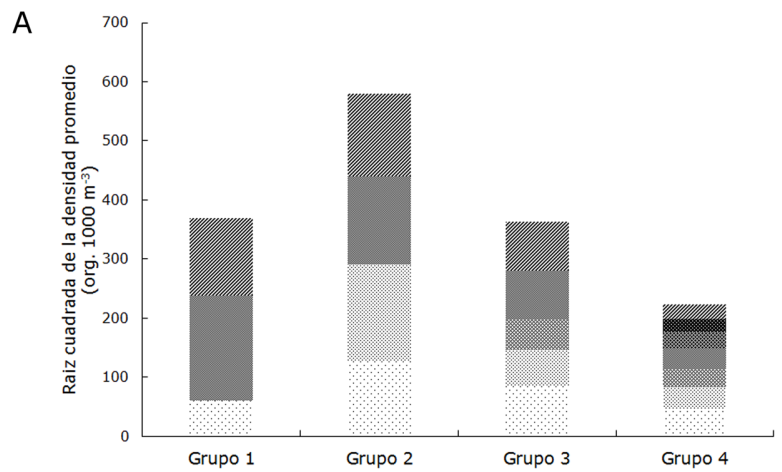

B
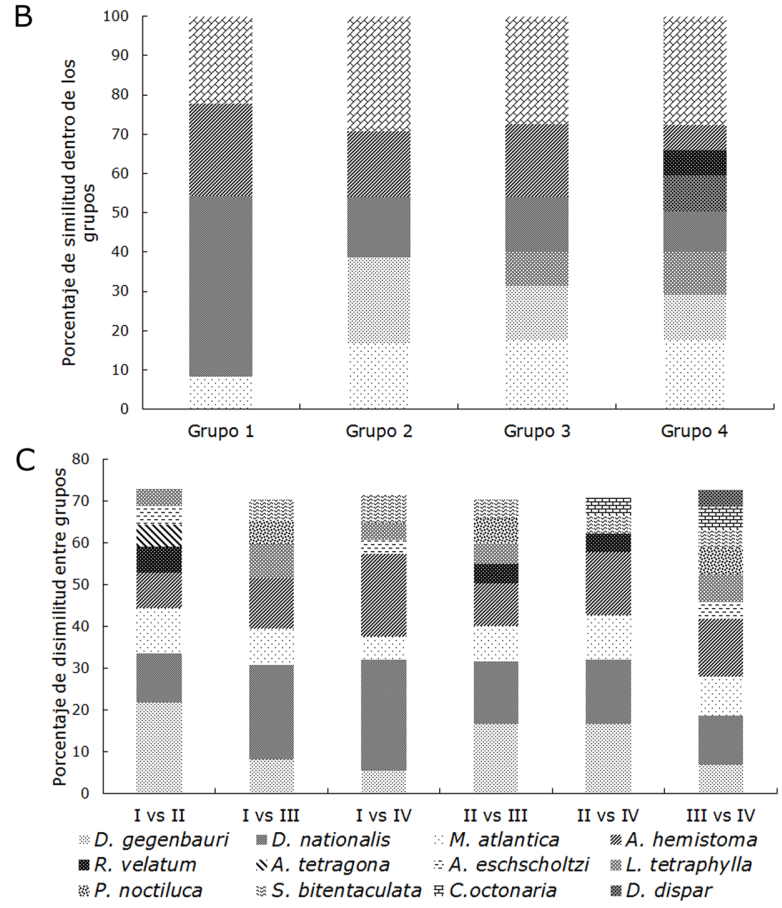

Figura 7. Análisis SIM PER mostrando la abundancia promedio de las especies (A), con los mayores porcentajes de contribución dentro de los grupos (B), así como los porcentajes de disimilitud entre ellos (C) / Simper analysis showing the average abundance of the species (A), with the highest similarity contribution within each group (B), as well as the percentages of dissimilarity between clusters (C) contribuyeron a la diferenciación entre grupos, como lo fueron Abylopsis eschscholtzi, Abylopsis tetragona, Cunina octonaria, Pelagia noctiluca y Solmundella bitentaculata (Fig. 7C).

Los primeros 2 ejes del análisis multivariado representaron el 78,42\% de la variación. Fue posible observar que existe una relación positiva entre la temperatura y el oxígeno disuelto y que dichas variables tienen una relación inversa con la salinidad y la clorofila. Las especies Agalma sp., Calycopsis simplex, Diphyes dispar, D. nationalis, Eudoxoides mitra, Iasis cylindrica, Lovenella clausa, Liriope tetraphylla, Nanomia bijuga, Pegea confoederata, Thalia democratica, Sphaerocoryne bedoti y $P$. noctiluca mostraron afinidad a los mayores registros de temperatura y oxígeno disuelto. El resto de las especies manifestaron mayor relación con las concentraciones elevadas de salinidad y clorofila (Fig. 8A). Los sitios de muestreo localizados en el sector sur del área de estudio, se caracterizaron por presentar mayores registros de temperatura y oxígeno disuelto, mientras que los más septentrionales por mostrar mayores registros de clorofila y salinidad (Fig. 8B).

\section{Discusión}

\section{VARIABLES Físicas, QUíMICAS Y BIOLÓGICAS}

Además de los factores generales que hacen variar las características hidrológicas de los océanos como la radiación solar y su ángulo de incidencia en el globo terrestre, también existen elementos regionales y/o locales que influyen en la temperatura del agua de los océanos, como lo es la presencia de corrientes de marinas y eventos de surgencia. Aunque no se realizó un análisis que permitiera determinar la influencia de las corrientes marinas en el área de estudio, posiblemente las menores temperaturas registradas en los sitios más septentrionales estuvieron influenciadas por la Corriente de California. Ya que, si bien el flujo de la Corriente de California se desvía hacía al oeste para alimentar a la Corriente Ecuatorial del Norte, existen corrientes geostróficas superficiales que fluyen hacia el sureste llegando a alcanzar los $13^{\circ} \mathrm{N}$ durante la primavera (Trasviña \& Barton 2008). No obstante, las condiciones ambientales en el Pacífico central mexicano, durante el período de recolecta, estarían especialmente determinadas por la influencia de la Corriente Occidental Mexicana (Fiedler \& Talley 2006, Kessler 2006), la cual, comienza a avanzar en mayo hacia la zona norte del área de estudio, transportando aguas cálidas, hasta alcanzar los $23^{\circ} \mathrm{N}$ en octubre (Trasviña \& 


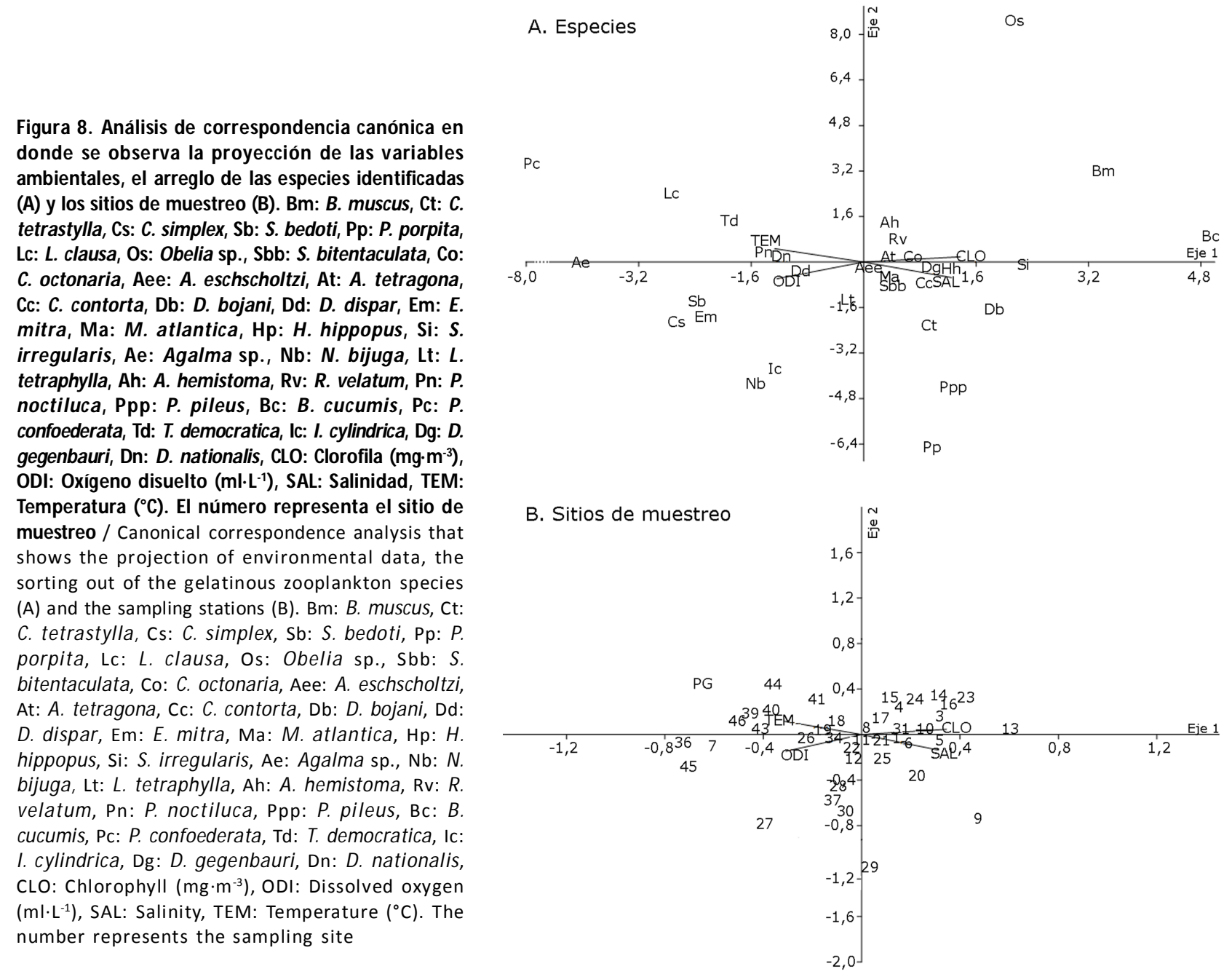

Andrade 2002). En el litoral de Jalisco y Colima, se ha observado que durante mayo se debilita la influencia de la Corriente de California habiendo un aumento de la temperatura superficial y hundimiento de la termoclina, reportándose temperaturas y salinidades promedio de $24,33^{\circ} \mathrm{C}$ y 34,59 respectivamente (Franco-Gordo et al. 2001). Particularmente los sitios de muestreo ubicados en la parte más meridional, presentarían temperaturas más elevadas debido a la presencia de una alberca de agua cálida, cuyo núcleo se encuentra ubicado frente a las costas de Michoacán y Guerrero, el cual mantiene temperaturas superficiales superiores a $\operatorname{los} 27^{\circ} \mathrm{C}$ (Trasviña \& Andrade 2002)

\section{COMPOSICIÓN DEL ZOOPLANCTON GELATINOSO}

Las medusas Cytaeis tetrastylla, Obelia sp., Solmundella bitentaculata, Cunina octonaria, Liriope tetraphylla, Aglaura hemistoma, Rhopalonema velatum y Pelagia noctiluca, ya habían sido reportadas con anterioridad en la zona de estudio (Foerster 1923, Segura-Puertas 1984, Segura-Puertas et al. 2010). Las especies Bougainvillia muscus y Porpita porpita representan nuevos registros para el Pacífico central mexicano, ya que únicamente habían sido reportadas en la costa Pacífica noroeste de México, el Golfo de México y/o el mar Caribe mexicano (Gómez 1991, Correia 1992, Suárez-Morales et al. 1999, Mendoza 2006, Calder \& Cairns 2009, Fernández-Álamo 2009). Las medusas Calycopsis simplex, Sphaerocoryne bedoti, Stauridiosarsia baukalion y Lovenella clausa 
se reportan por primera vez en aguas mexicanas (SeguraPuertas et al. 2003). Recientemente se identificaron 23 especies de medusas en la costa de los estados de Jalisco y Colima (Segura-Puertas et al. 2010), es decir, una mayor cantidad de especies a la encontrada en este trabajo (14 especies). La diferencia podría atribuirse a la ubicación de los sitios de recolecta, a la época climática y al tipo de muestreo realizado (oblicuo), así como a las características de la red empleada durante la captura del zooplancton (diámetro de boca de $60 \mathrm{~cm}, 300 \mathrm{~cm}$ de longitud y $500 \mu \mathrm{m}$ de abertura de malla). El muestrear a finales de la época de secas, así como el incluir sitios de recolecta oceánicos en lugar de únicamente costeros, podría resultar en una menor riqueza específica (Gaitán 2004, Zavolokin 2010, Rodríguez-Sáenz et al. 2012). Considerando que en la época de lluvias existe un mayor aporte de nutrientes proveniente de cuerpos de agua epicontinentales, es posible inferir que existe un aumento en el número de productores primarios especialmente en un radio cercano a los sitios de descarga, lo que podría favorecer la proliferación de una mayor cantidad de especies (Matsumura-Tundisi et al. 1997, D'Croz et al. 1999).

En aguas del Pacífico mexicano se han registrado 73 especies de sifonóforos, siendo las regiones de Baja California y/o el Golfo de California las áreas más estudiadas (Gasca 2002). Se reporta por primera vez en aguas del Pacífico central mexicano a la especie Abylopsis tetragona, que junto con Abylopsis eschscholtzi habitan las mismas regiones del Pacífico (Bigelow 1911, Alvariño 1971, 1972). El hallazgo de Sphaeronectes irregularis constituye un nuevo registro para aguas mexicanas, dicho sifonóforo ha sido reportado en el mar Mediterráneo y en otras zonas del océano Pacífico (Bouillon et al. 2004, Palma $\&$ Apablaza 2004). De manera general, la riqueza registrada de sifonóforos podría considerarse baja, algo que usualmente puede atribuirse a la selectividad del método de recolecta empleado (Gasca 2002).

Las únicas especies de ctenóforos identificadas en el presente estudio fueron Beroe cucumis y Pleurobrachia pileus, esta última registrada previamente en el puerto de Acapulco (Bigelow 1912). No se encontraron más registros de ctenóforos en el Pacífico tropical mexicano; sin embargo, Gómez (1991) incluyó 2 especies (Pleurobrachia bachei y Beroe sp.) en estuarios del noroeste de México. Las dificultades para la determinación taxonómica de estos ejemplares, se centran principalmente en los métodos de fijación y/o conservación, por lo que seguramente se subestima la riqueza y/o abundancia de este grupo (Sullivan \& Gifford 2009).
En Baja California, Hereu et al. (2006) identificaron 25 especies de salpas, entre ellas, Iasis cylindrica, Thalia democratica y Pegea confoederata, reportando un máximo de 17 especies en invierno y un mínimo de 5 en primavera.

De manera general las especies observadas tienen amplia distribución en zonas tropicales (Alvariño \& Leira 1986, Hereu et al. 2006, Morales-Ramírez \& Nowaczyk 2006, Segura-Puertas et al. 2010). Sin embargo, debido a la carencia de trabajos de este tipo, los estudios en zonas costeras usualmente suelen revelar nuevos registros. Palma \& Apablaza (2004) señalaron la presencia de 44 especies de zooplancton gelatinoso (23 especies de sifonóforos, 17 de hidromedusas y 4 de ctenóforos) en el norte del Sistema de la Corriente de Humboldt. En este caso, la mayor riqueza específica podría atribuirse a las variaciones de los parámetros hidrológicos, a los eventos de surgencia costera en su zona de estudio y a que obtuvieron muestras de zooplancton tanto de día como de noche. En la región del Golfo Dulce, Pacífico de Costa Rica, se registraron 41 especies, correspondientes a 33 especies de cnidarios (17 hidromedusas y 16 sifonóforos), 2 ctenóforos y 6 taliáceos (Morales-Ramírez \& Nowaczyk 2006). La riqueza de ctenóforos y taliáceos son similares a los de este estudio, y mayor en el caso de las hidromedusas y sifonóforos. Estas diferencias pueden radicar en la época de muestreo, la ubicación de los puntos de recolecta con respecto a la línea de costa, así como el hecho de que el trabajo de Morales-Ramírez \& Nowaczyk (2006) se llevó a cabo en un evento de El Niño, fenómeno usualmente relacionado con un incremento de especies tropicales (Aronés-Flores \& Ayón-Dejo 2002). En la región de la Bahía de Panamá, Miglietta et al. (2008), durante 113 días de recolección de hidromedusas en períodos de surgencia y no surgencia identificaron 7, 9 y 6 taxones a nivel de especie, género y orden, respectivamente. Observaron que la composición de las especies fue constante a lo largo del período de muestreo, con el registro de únicamente 2 ejemplares propios de eventos de surgencia. Por otro lado, reportaron aumentos significativos en la densidad de medusas durante los eventos de surgencia. Por su parte, Baldrich \& López (2013) encontraron 18 especies de hidromedusas en el Pacífico colombiano, 9 de ellas meroplanctónicas y 9 holoplanctónicas. Dicho autores mencionan, que el bajo número de especies meroplanctónicas no implica la ausencia de organismos en el medio, ya que podrían estar presentes en su forma bentónica o en un estado latente de resistencia. 
Frecuencia, DENSIDAD, EQUidad Y DIVERSIDAD DEL ZOOPLANCTON GELATINOSO

Las medusas con las mayores frecuencias de aparición en el área de estudio pertenecen a las subclases Trachymedusae y Narcomedusae. Estos organismos usualmente son encontrados con una alta frecuencia de aparición, ya que suelen estar bien representadas tanto en aguas oceánicas como costeras (Segura et al. 2010). El sifonóforo Muggiaea atlantica fue la especie que presentó la mayor frecuencia de aparición, estando únicamente ausente en un sitio de muestreo, corroborando la gran dispersión que puede tener esta especie en distintas regiones del Pacífico tropical (Gasca 2002, Morales-Ramírez \& Nowaczyk 2006).

Esnal \& Simone (1982) registraron a Doliolum nationalis como la especie de doliólido más frecuente en el Golfo de México y mar Caribe, seguida por Doliolum denticulatum, Doliolina (Doliolina) mulleri y Dolioletta gegenbauri. Por otro lado, Hereu et al. (2006) registraron una aparición continua de Thalia democratica y Thalia orientalis en distintos periodos de muestreo en Baja California, México. Las especies más abundantes del zooplancton gelatinoso en el área de estudio fueron $D$. gegenbauri y $D$. nationalis, que en conjunto representaron el $51 \%$ de la densidad total. Las mayores densidades de estos organismos se encontraron en la zona costera, coincidiendo con su tendencia a ser más abundantes en zonas litorales (Apablaza \& Palma 2005). Estos organismos son un componente sumamente importante de la biomasa del zooplancton herbívoro en las capas superficiales de los mares tropicales, ya que son capaces de colonizar zonas donde las condiciones son adecuadas a un mayor ritmo que el de otros zoopláncteres (Andersen 1998). En el caso de las salpas, una vez superada la densidad de 1000 org. $1000 \mathrm{~m}^{-3}$, es posible considerarlo un agrupamiento (Hereu et al. 2006). Conforme a dicho criterio, se encontraron agrupamientos de T. democratica (E36: 3000 org. $1000 \mathrm{~m}^{-3}$; E43: 2000 org. $1000 \mathrm{~m}^{-3}$ ), Iasis cylindrica (E29: 5000 org. $1000 \mathrm{~m}^{-3}$ ) y Pegea confoederata (PG: 1000 org. $1000 \mathrm{~m}^{-3}$ ).

Las medusas con mayores densidades en este estudio, ya habían sido previamente registradas como las especies más abundantes en el Pacífico mexicano, ya que se trata de especies generalistas, cosmopolitas y/o de amplia distribución (Segura-Puertas 1984, Segura-Puertas et al. 2010). El sifonóforo $M$. atlantica fue otro de los cnidarios que presentó altas densidades, siendo reportado en otros sitios del Pacífico oriental como un componente importante de la densidad (Palma \& Apablaza 2004).
En la estación 42, un sitio ubicado a 6 mn de la línea de costa, se encontraron altas densidades de taliáceos y bajas concentraciones de clorofila, reforzando la idea de que estos organismos pueden consumir en gran parte o en su totalidad la producción primaria diaria (Hereu et al. 2006). La máxima densidad de zooplancton gelatinoso se registró en dicha estación, en donde las especies Aglaura hemistoma, D. gegenbauri, D. nationalis, Nanomia bijuga, Obelia sp., Pleurobrachia pileus y Stauridiosarsia baukalion mostraron su máxima densidad.

La especie más abundante en este estudio fue $D$. gegenbauri que aportó un $61 \%$ de la densidad total del punto de muestreo 42. En un evento de agrupamiento en la Corriente del Golfo se registraron densidades promedio de $728000 \pm 474000$ org. $1000 \mathrm{~m}^{-3}$, densidades mínimas de 14000 org. $1000 \mathrm{~m}^{-3}$ y máximas de 3216000 org. $1000 \mathrm{~m}^{-3}$ (Deibel 1985). Por lo que, los valores registrados en la estación 42 nos indican que durante el periodo de muestreo $D$. gegenbauri estaban formando un agrupamiento.

La menor densidad de organismos se encontró en el sitio 19, ubicado en la zona oceánica del área de estudio. Dicha provincia del océano Pacífico mexicano ha sido señalada como oligotrófica (López-Calderón et al. 2006). Por lo que esa condición, pudo haberse reflejado en bajas concentraciones de organismos.

En el presente trabajo se registraron altas densidades de organismos. Algunas especies como A. hemistoma y Pelagia noctiluca ya han sido reportadas anteriormente en núcleos de concentraciones mayores a 10000 org. 1000 $\mathrm{m}^{-3}$ en el Pacífico tropical (Segura-Puertas 1984). Las condiciones oceanográficas registradas en el año 2011 sugieren que se trata de un año sutilmente influenciado por el fenómeno 'La Niña' con tendencia a la neutralidad de las condiciones oceánicas (ENSO-neutral). Si bien la respuesta a este fenómeno ocurre a nivel específico, las condiciones acarreadas por este evento pudieron haber favorecido la elevada abundancia de organismos.

Con respecto a la diversidad de Shannon, en el sector norte del área de estudio se han registrado diversidades promedio de 1,00 bit para la comunidad de medusas (Segura et al. 2010). Mientras que en la Bahía de Panamá se han reportado valores máximos de 1,65 bits (Baldrich \& López 2013). Los resultados de la diversidad obtenidos en el presente trabajo, fueron mayores a los mencionados anteriormente, lo cual se atribuye a que se consideraron otros grupos taxonómicos. La equidad de Pielou alcanzó su máximo valor en la estación de muestreo 7 , en donde la especie $P$. noctiluca presentó una alta dominancia que afectó la biomasa zooplanctónica de los demás grupos (Cataletto et al. 1995). 


\section{Análisis de la COMUNiDAd}

A pesar de que el porcentaje de contribución de los taxones cambió en los grupos formados, fueron las mismas 4 especies las que en su conjunto determinaron la formación de los grupos. Este suceso se podría ver favorecido por la estrechez de la plataforma continental en el Pacífico central mexicano. Lo cual, permite que exista una menor heterogeneidad en los parámetros oceanográficos en el área de estudio, facilitando la presencia de especies oceánicas en aguas neríticas. Por lo que, sería común que unas pocas especies sean la principal fuente de contribución a la similitud espacial (Suárez-Morales et al. 2000, Valencia et al. 2013).

La participación de las especies que contribuyen en menor grado a la formación de los grupos podría estar limitada por la baja capacidad de dispersión y por las necesidades fisiológicas que presentarían estos individuos, limitándolos a distribuirse en un área más reducida. De esta manera, la presencia y distribución de las especies y por ende su porcentaje de contribución a la formación de grupos, estaría determinada por una serie de interacciones biológicas y condiciones ambientales, como los son procesos oceanográficos de convergencias, divergencias, frentes, remolinos y surgencias (Baldrich \& López 2013).

Las especies afines a los mayores valores de salinidad y clorofila mostraron sus mayores densidades en la zona norte del área de estudio. La mayoría de ellas se agrupó en el conjunto II del análisis de similitud, apareciendo en sitios costeros, en los cuales el lance de la red se realizó a profundidades menores a $\operatorname{los} 75 \mathrm{~m}$. Es sabido que normalmente la densidad de los seres del zooplancton está relacionada con la disponibilidad de alimento, por lo que, la alta productividad pudo haber favorecido la abundancia de organismos (Baldrich \& López 2013).

\section{Agradecimientos}

Este trabajo es parte de la tesis presentada por EGM para obtener el título de Biólogo en la Universidad Michoacana de San Nicolás de Hidalgo. El tiempo de buque para la campaña oceanográfica MareaR-III realizada a bordo del B/O 'El Puma' fue financiado por la Universidad Nacional Autónoma de México; se agradece el apoyo de David Uriel Hernández Becerril, jefe de la campaña oceanográfica. Agradecemos a Lucio Loman Ramos por corroborar la identificación de las medusas y a Eduardo Suárez Morales por los comentarios durante la redacción del documento.

\section{LITERATURA CITADA}

Agassiz Á \& AG Mayer. 1902. Reports on the scientific results of the Expeditions to the Tropical Pacific, ALBATROSS, 1899-1900. III Medusae. Bulletin of the Museum of Comparative Zoology Harvard College 26: 137-176.

Alvarez CG, H Mianzan \& A Madirolas. 2003. Acoustic characterization of gelatinous plankton aggregations: four case studies from the Argentine continental shelf. Journal of Marine Science 60(3): 650-657.

Alvariño Á. 1971. Siphonophores of the Pacific with a review of the world distribution. Bulletin of the Scripps Institution of Oceanography, University of California 16: 1-432.

Alvariño Á. 1972. Zooplancton del Caribe, Golfo de México y regiones adyacentes del Pacífico. Memorias del IV Congreso Nacional de Oceanografía, México: 223-247.

Alvariño Á \& M Leira. 1986. El zooplancton del Pacífico ecuatoriano. Investigaciones Marinas CICIMAR 3(1): 69110 .

Andersen V. 1998. Salp and pyrosomid blooms and their importance in biogeochemical cycles. In: Bone Q (ed). The biology of pelagic tunicates, pp. 125-138. Oxford University Press, Oxford.

Apablaza P \& S Palma. 2005. Primer registro de Dolioletta gegenbauri (Uljanin, 1884) y Doliolum nationalis Borgert, 1893 en aguas chilenas (Tunicata, Doliolida). Investigaciones Marinas 33(1): 127-130.

Aronés-Flores K \& P Ayón-Dejo. 2002. Effects of El Niño 19971998 on copepod community structure at San José coastal station (Peru). Investigaciones Marinas 30(1): 104-105.

Bailey TG, MJ Youngbluth \& GP Owen. 1995. Chemical composition and metabolic rates of gelatinous zooplankton from midwater and benthic boundary layer environments off Cape Hatteras, North Carolina, USA. Marine Ecology Progress Series 122: 121-134.

Baldrich AM \& RH López. 2013. Hidromedusas del pacífico colombiano: aspectos básicos de su dinámica ecológica. Revista Facultad de Ciencias Básicas 9(1): 108-131.

Bigelow HB. 1909. Reports on the scientific results of the Expedition to the Eastern Tropical Pacific of 'Albatross', from October, 1904 to March 1905. Bulletin of the Museum of Comparative Zoology Harvard College 37: 1-245.

Bigelow HB. 1911. The Siphonophorae. Eastern Tropical Pacific Expedition ALBATROSS. Bulletin of the Museum of Comparative Zoology Harvard College 38(2): 173-401.

Bigelow HB. 1912. Report of the scientific research expedition to the Eastern Tropical Pacific. The ctenophores. Bulletin of the Museum of Comparative Zoology Harvard College 54(12): 369-408.

Bouillon J, MD Mendel, F Pagés, JM Gili, F Boero \& C Gravili. 2004. Fauna of the Mediterranean Hydrozoa. Scientia Marina 68(2): 5-438. 
Calder DR \& SD Cairns. 2009. Hydroids (Cnidaria: Hydrozoa) of the Gulf of Mexico. In: Felder DL \& DK Camp (eds). Gulf of Mexico-Origins, waters, and biota. Vol. I: Biodiversity: 381-394. Texas A \& M Press, Texas.

Cataletto B, E Feoli, SF Umani \& S Cheng-Yong. 1995. Eleven years of time-series analysis on the net-zooplankton community in the Gulf of Trieste. ICES Journal of Marine Science 52(3-4): 669-678.

Clark KR \& RM Warwick. 2001. Change in marine communities: an approach to statistical analysis and interpretation, 176 pp. PRIMER-E, Plymouth.

Clarke KR \& RN Gorley. 2006. PRIMER v6: User Manual/ Tutorial, 190 pp. PRIMER-E, Plymouth.

Collins NR \& R Williams. 1981. Zooplankton of the Bristol Channel and Severn Estuary. The distribution of four copepods in relation to salinity. Marine Biology 64(3): 273-283.

Correia VK. 1992. Medusas del Golfo de México y litoral norte/noreste de Brasil (ocurrencia y distribución). Tesis Doctoral. Facultad de Ciencias, Universidad Nacional Autónoma de México, México, 284 pp.

D'Croz L, D Ross-Robertson \& JA Martínez. 1999. Crossshelf distribution of nutrients, plankton, and fish larvae in the San Blas Archipelago, Caribbean Panama. Revista de Biología Tropical 47(1-2): 203-215.

Deibel D. 1985. Blooms of the pelagic tunicate, Dolioletta gegenbauri: Are they associated with Gulf Stream frontal eddies? Journal of Marine Research 43: 211-236.

Esnal GB \& LC Simone. 1982. Doliolidos (Tunicata, Thaliacea) del Golfo de México y mar Caribe. Physis (Buenos Aires) Sección A 40: 51-57.

Fagetti E. 1973. Medusas de aguas chilenas. Revista de Biología Marina 15(1): 31-75.

Fernández-Álamo MA. 2009. Cnidarios, poliquetos, crustáceos (decápodos) y quetognatos pelágicos del Golfo de California. Informe Final del Proyecto DJ036 SNIBCONABIO: $1-23$.

Fiedler PC \& LD Talley. 2006. Hydrography of the eastern tropical Pacific: a review. Progress in Oceanography 69(2): 143-180.

Foerster RE. 1923. The hydromedusae of the west coast of North America with special reference to those of the Vancouver Island region. Tesis de Maestría, Deparment of Zoology, University of British Columbia, Vancouver, 136 pp.

Franco-Gordo C, E Suárez-Morales, E GodínezDomínguez \& R Flores-Vargas. 2001. A seasonal survey of the fish larvae community of the central Pacific coast of Mexico. Bulletin of Marine Science 68(3): 383-396.

Gaitán EN. 2004. Distribución, abundancia y estacionalidad de Liriope tetraphylla (Hidromedusa, Trachymedusae) en el Océano Atlántico Sudoccidental y su rol ecológico en el estuario del Río de la Plata. Tesis de Licenciatura, Facultad Ciencias Exactas y Naturales, Universidad Nacional de Mar de Plata, Mar de Plata, 42 pp.
Gasca R. 2002. Lista faunística y bibliografía comentadas de los sifonóforos (Cnidaria: Hydrozoa) de México. Anales del Instituto de Biología, Universidad Nacional Autónoma de México, Serie Zoología 73(2): 123-143.

Godeaux J. 1998. The relationships and systematics of the Thaliacea, with keys for identification. In: Bone Q (ed). The biology of pelagic tunicates, pp. 273-294. Oxford University Press, Oxford.

Gómez AS. 1991. Contribución al estudio faunístico de celenterados y ctenóforos del plancton estuarino del noroeste de México. Anales del Instituto de Biología, Universidad Nacional Autónoma de México, Serie Zoología 62(1): 1-10.

Hereu CM, BE Lavaniegos, G Gaxiola \& MD Ohman. 2006. Composition and potential grazing impact of salps assemblage off Baja California during the 1997- 1999 El Niño and La Niña. Marine Ecology Progress Series 318: 123-140.

Hereu CM, BE Lavaniegos \& R Goericke. 2010. Grazing impact of salp (Tunicata, Thaliacea) assemblages in the eastern tropical North Pacific. Journal of Plankton Research 32(6): 785-804.

Kessler WS. 2006. The circulation of the eastern tropical Pacific: a review. Progress in Oceanography 69: 181-217.

Lebrato M \& DOB Jones. 2011. Expanding the oceanic carbon cycle: Jellyfish biomass in the biological pump. Biochemist e-volution: $35-39$.

Licea DS. 1974. Sistemática y distribución de las diatomeas de la laguna de Agiabampo, Son./Sin. México. Anales del Instituto de Ciencias del Mar y Limnología, Universidad Nacional Autónoma de México 1(1): 99-156.

López-Calderón J, H Manzo-Monroy, E Santamaría-delÁngel, R Castro, A González-Silvera \& R MillánNúñez. 2006. Variabilidad de mesoescala del Pacífico tropical mexicano mediante datos de los sensores TOPEX y SeaWiFS. Ciencias Marinas 32(3): 539-549.

Lucas C. 2011. Los servicios e impactos de las medusas a la sociedad. VI Debate sobre biología de la conservación: el papel global de las medusas y su aumento en el océano, Madrid. <http://www.fbbva.es/TLFU/dat/ programa_debate _conservacion_biodiversidad_2011.pdf

Maas O. 1897. Die Medusen. Reports on an exploration off the west coast of Mexico, Central and South America, and off the Galapagos Islands by the U.S. Steamer 'Albatross' during 1891. Bulletin of the Museum Comparative of Zoology Harvard College 23: 1-92.

Madin LP, P Kremer, PH Wiebe, JE Purcell, EH Horgan \& DA Nemazie. 2006. Periodic swarms of the salp Salpa aspera in the Slope Water off the NE United States: biovolume, vertical migration, grazing, and vertical flux. Deep-Sea Research I 53: 804-819.

Matsumura-Tundisi T. 1997. Composition and vertical distribution of zooplankton in Lake Dom Helvécio MG, Brazil. In: Tundisi JG \& Y Saijo (eds). Limnological studies on the Rio Doce Valley Lakes, Brazil, pp. 309-326. Academia Brasileira de Ciencias, Rio de Janeiro. 
Mendoza BMA. 2006. Diversidad de medusas (Phylum: Cnidaria) en la Laguna Madre, Tamaulipas. Tesis de Licenciatura, Escuela Nacional de Ciencia Biológicas, Instituto Politécnico Nacional, México, 61 pp.

Mianzan H. 1999. Ctenophora. In: Boltovskoy D (ed). South Atlantic zooplankton, pp. 561-573. Backhuys Publishers, Leiden.

Miglietta MP, M Rossi \& R Collin. 2008. Hydromedusa blooms and upwelling events in the Bay of Panama, Tropical East Pacific. Journal of Plankton Research 30(7): 783-793.

Morales-Ramírez A \& J Nowaczyk. 2006. El zooplancton gelatinoso del Golfo Dulce, Pacífico de Costa Rica, durante la transición de la estación lluviosa a la seca 1997-1998. Revista de Biología Tropical 54(1): 201-223.

Palma S \& P Apablaza. 2004. Abundancia estacional y distribución vertical del zooplancton gelatinoso carnívoro en un área de surgencia en el norte del sistema de la Corriente de Humboldt. Investigaciones Marinas 32(1): 49-70.

Pugh PR. 1984. The diel migrations and distributions within a mesopelagic community in the north east Atlantic. 7. Siphonophores. Progress in Oceanography 13(3): 461-489.

Purcell JE. 1985. Predation on fish eggs and larvae by pelagic cnidarians and ctenophores. Bulletin of Marine Science 37(2): 739-755.

Purcell JE, S Uye \& WT Lo. 2007. Anthropogenic causes of jellyfish blooms and their direct consequences for humans: a review. Marine Ecology Progress Series 350: 153-174.

Rodríguez-Sáenz K, JA Vargas-Zamora \& L SeguraPuertas. 2012. Medusas (Cnidaria: Hydrozoa) de una zona de afloramiento costero, Bahía Culebra, Pacífico, Costa Rica. Revista de Biología Tropical 60(4): 1731-1748.

Sánchez MP \& ME Ponce. 1996. Métodos hidrobiológicos II. Estudio y colecta de organismos marinos, estuarinolagunares y de agua dulce, 208 pp. Universidad Autónoma Metropolitana, México.

Schiariti A. 2008. Historia de vida y dinámica de poblaciones de Lychnorhiza lucerna (Scyphozoa) ¿Un recurso pesquero alternativo? Tesis Doctoral, Facultad de Ciencias Exactas y Naturales, Universidad de Buenos Aires, Buenos Aires, 209 pp.

Segura-Puertas L. 1984. Morfología, sistemática y zoogeografía de las medusas (Cnidaria: Hydrozoa y Scyphozoa) del Pacífico tropical oriental. Instituto de Ciencias del Mar y Limnología, Universidad Nacional Autónoma de México, Publicación Especial 8: 1-320.
Segura-Puertas LE, E Suárez-Morales \& L Celis. 2003. A checklists of the Medusae (Hydrozoa, Scyphozoa and Cubozoa) of Mexico. Zootaxa 194: 1-15.

Segura-Puertas L, C Franco-Gordo, E Suárez-Morales, R Gasca \& E Godínez-Domínguez. 2010. Summer composition and distribution of the jellyfish (Cnidaria: Medusozoa) in the shelf area off the central Mexican Pacific. Revista Mexicana de Biodiversidad 81: 103-112.

Suárez-Morales E, L Segura-Puertas \& R Gasca. 1999. A survey of the reef-related medusa (Cnidaria) community in the western Caribbean Sea. Gulf Research Reports 11:2331.

Suárez-Morales E, C Franco-Gordo \& M Saucedo. 2000. On the pelagic copepod (Crustacea: Copepoda) community of the central Mexican tropical Pacific (Autumn, 1990). Crustaceana 73: 751-761.

Sullivan LJ \& DJ Gifford. 2009. Preservation of the larval ctenophore Mnemiopsis leidyi A. Agassiz (Ctenophora, Lobata). Journal of Plankton Research 31: 921-926.

Touzri C, H Hamdi, J Goy, D Yahia \& N Mohamed. 2012. Diversity and distribution of gelatinous zooplankton in the Southwestern Mediterranean Sea. Marine Ecology 33(4): 393-406.

Trasviña A \& CA Andrade. 2002. La circulación costera del Pacifico Tropical Oriental, con énfasis en la Alberca Cálida Mexicana (ACM). En: Circulación oceánica y climatología tropical en México y Colombia. Diálogo entre Pares 1, Consejo Nacional de Acreditación-Ministerio de Educación. Corcas Editores, Bogotá, pp. 9-37.

Trasviña A \& ED Barton. 2008. Summer circulation in the Mexican tropical Pacific. Deep Sea Research I: Oceanographic Research Papers 55(5): 587-607.

Valencia B, B Lavaniegos, A Giraldo \& E Rodríguez-Rubio. 2013. Temporal and spatial variation of hyperiid amphipod assemblages in response to hydrographic processes in the Panama Bight, eastern tropical Pacific. Deep Sea Research Part I: Oceanographic Research Papers 73: 46-61.

Zavolokin AV. 2010. Distribution and abundance dynamics of jellyfish in the Sea of Okhotsk. Russian Journal of Marine Biology 36(3): 157-166. 